\title{
Towards New States of Matter
}

\author{
Fábio L. Braghin \\ Instituto de Física, Universidade de São Paulo, C.P. 66.318, CEP 05315-970, São Paulo, SP, Brazil
}

Received on 9 September, 2003

\begin{abstract}
A linear realization of flavor $S U(3) \times S U(3)$ symmetry is developped with scalar and pseudoscalar mesons coupled to baryons and massive vector fields. This system may be also coupled to colored quarks and gluons, which are expected to be deconfined above a critical range of energy densities and seemingly inside baryons. The coupling to vector fields may lead to finite density baryonic and also anti-baryonic matter. Prescriptions for the stability equation which yields bound systems is verified. A qualitative discussion of the possible resulting (QCD - type) phase diagram is discussed including the possibility of finding several kinds of superfluid (and eventually superconductive) states. Some bosonic fields are assumed to have non zero expected values, at least at low energies, corresponding to dynamical symmetry breakings. A generalized symmetry radius is found and the masses of the particles are found to vary with density. Possible relevance to relativistic heavy ion collision is pointed out.
\end{abstract}

\section{Introduction and $S U(3)$ Linear Sigma Model}

The fundamental theory for strong interacting systems is quantum chromodynamics (QCD), which is expected to lead to the description of nuclear interacting systems. It exhibits the phenomenum of color confinement, containing a non abelian color structure, and a flavor-chiral structure (in particular for lightest hadrons) which remains at the hadronic level. However it seems to be fair to ask the following questions: is QCD a theory for one interaction? or: does it provide an unifying picture of two fundamental interactions (of flavor and color)? For the light hadronic particles, chiral symmetry $(S U(2) \times S U(2))$ yields a convenient framework for classifying the hadrons and describing low energy processes. Chiral symmetry is expected to be spontaneously broken down to isospin in the Nambu realization. It yields an intrincated vacuum with a scalar (quark-anti-quark) condensate [1]. It has been difficult to describe nuclear interactions departing from such picture with many attempts which have hardly achived small part of this [2]. Furthermore and as a consequence the phase diagram of matter is not completely known.

In a series of papers I am proposing an unifying frame for the development of strong interacting physics at finite baryonic (and eventually quark) densities. This is built over properties of hadrons expected to have grounds on QCD. In this work, which is nearly a resumé of part of them, it is discussed the spontaneous breakdown of flavor and chiral symmetries, and speculations on existence of anti-matter bound states. For this the $S U(3) \times S U(3)$ symmetry is considered in an effective linear sigma model with baryons, (pseudo+)scalar mesons, vector mesons and fields, which can be coupled to deconfing quarks and gluons at high energy densities, above asymptotic freedom scales. Properties of the hadrons at finite hadronic densities are described by "condensates" of bosonic fields. Phase diagram of strong interactions would include several superfluid/superconductive states. It may contain a further axis of anti-baryonic density which may be a third (in)dependent axis in this diagram or not [8].

The $S U(3) \times S U(3)$ invariant Lagrangian density of the Linear Sigma Model with three-component baryons, $N_{i}(\mathbf{x})$, scalars ${ }^{1}$, pseudoscalars mesons and vector fields. The spin zero mesons are denoted by $(\Sigma, \Pi)$ respectively as nonets defined below. These bosons, as well as the baryons, were covariantly coupled to (massive) gauge isovector-vector fields $V_{\mu}, \vec{\rho}_{\mu}$ and $\left(\vec{A}_{1}\right)_{\mu}(S U(2) \times S U(2))$. The Lagrangian density reads:

$$
\begin{aligned}
& \mathcal{L}=\bar{N}_{i}(\mathbf{x})\left(i \gamma_{\mu} \mathcal{D}_{t}^{\mu}-g_{s}\left(\Sigma+i \gamma_{5} . \Pi\right)\right) N_{i}(\mathbf{x})+\frac{1}{2}\left(\partial_{\mu} \Sigma . \partial^{\mu} \Sigma+\partial_{\mu} \Pi . \partial^{\mu} \Pi\right)+\frac{\mu^{2}}{2} \operatorname{Tr}|\Sigma+i \Pi|^{2}+ \\
& -\frac{1}{4} F_{\mu \nu} F^{\mu \nu}-\lambda_{1} \operatorname{Tr}\left(|(\Sigma+i \Pi)|^{2}\right)^{2}-\lambda_{2}\left(\operatorname{Tr}\left(|\Sigma+i \Pi|^{2}\right)\right)^{2}+\frac{1}{2} m_{V}^{2} V_{\mu} V^{\mu}+\mathcal{L}_{\rho}+\mathcal{L}_{A_{1}}+\mathcal{L}_{q, g, i n t}
\end{aligned}
$$

\footnotetext{
${ }^{1}$ Scalars are being found and discussed in the literature and may form a quark and anti-quark nonet or octet, and/or may be multi-quark states with gluonic components or not [?]
} 
where the last three terms stand for the "gauge" isovector and axial fields, in the $S U(2) \times S U(2)$ chiral model, are the rho and $A_{1}$ and quark and gluon terms with interactions also with hadrons which would be relevant for energy densities close to those of the deconfining phase transition with asymptotic freedom. Therefore these quark and gluon terms were not considered in the calculation. The covariant derivative is: $\mathcal{D}_{t}^{\mu}=\partial^{\mu}-i g_{V} V^{\mu}-i g_{3}\left(A_{L}^{\mu}-A_{R}^{\mu}\right)$, with the "generalized right and left" combinations (in the SU(2): $\left.A_{R, L}^{\mu} \propto A_{\rho}^{\mu} \pm A_{A_{1}}^{\mu}\right)$ of the flavor-chiral partner isovector mesons [1]. The scalar (pseudoscalar) matrix was chosen to be written as: $\Sigma=\sum_{a} \lambda_{a} \sigma_{a}\left(\Pi=\sum_{a} \lambda_{a}\right.$ " $\pi_{a}$ ") where the $\lambda_{a}$ are the Gell-Mann matrices in the adjoint representation and $\sigma_{a}$ (" $\pi_{a}$ ") the scalar (pseudoscalar) fields. The extension of these ideas of spontaneously broken chiral-flavor symmetries in more complete frama of $S U\left(N_{f}\right) \times S U\left(N_{f}\right)$ ( $\left.N_{f} / g t 3\right)$ is being planned.

The minimization of the spin zero potential for the $S U(3)$ symmetric limit results in a strange-chiral radius $v_{3}$ that can be defined with a "mixed" coupling: $\lambda_{3}=$ $\lambda_{2}+\lambda_{1} / 2$, reading: $v_{3}^{2}=\mu^{2} /\left(4 \lambda_{3}\right)$, where $\mu^{2}$ is the mass from the Lagrangian. This is the analogous to the chiral radius in $S U(2) \times S U(2)$. The Lagrangian term would be written as: $-\lambda_{3}\left(\operatorname{Tr}\left|\Sigma^{2}+\Pi^{2}\right|-v_{3}^{2}\right)^{2}$.

\section{Characterizing Some States}

The averaged energy density is calculated for quantized baryons initially considered to form a Fermi liquid and all bosonic variables are condensated states, for the spin zero bosons the quantum fluctuations taken into account with a variational approach with Gaussian wavefunctionals [4, 3], such that the averaged energy reads:

$$
\mathcal{H}^{t o t}=\rho_{f}+g_{V} V_{0} \rho_{B}-\frac{1}{2} \tilde{m}_{V}^{2} V_{0}^{2}+\lambda_{3}\left(T r\left|\tilde{\Sigma}^{2}+\tilde{\Pi}^{2}\right|-\tilde{v}_{3}^{2}\right)^{2}
$$

Where $\tilde{m}$ is an "effective mass" for the vector field (temporal component) and $\tilde{v}_{3}$ a modified chiral radius due to quantum fluctuations (also considered to shift the spin zero fields classical values with tilde) which are not calculated explicitely. We perform a truncation of the effective potential which corresponds to: 1) equate all the quantum two point functions which shift the classical values, 2) neglect further terms in the above expression of the energy density, as proposed in [4]. ¿From now on, $\rho_{B}$ (and eventually $\left.\rho_{f}\right)$ are to depend on the classical values of spin zero variables (like $\tilde{\sigma}, \tilde{\boldsymbol{\pi}}$ ) and $V_{0}$, as variational parameters. Correlations and many-body effects of the fermions for the bosonic fields are considered such that the baryonic density depend on them and vice versa. ¿From this consideration the equations of the system are derived [4].

The stability condition of the bound matter system can be written as:

$$
\frac{d \mathcal{H}}{d \rho_{B}}=\left.\frac{\mathcal{H}}{\rho_{B}}\right|_{\rho_{B}=\rho_{0}}=-\frac{E}{A}<0 .
$$

Where $-E / A$ is the binding energy per baryon ${ }^{2}$. To look for solution for this expression we consider that each kind of hadrons satisfy it nearly independently [4]. A solution for this prescription [4] for the fermion density which reproduces usual values for $\rho_{f}$ is given by [4]:

$$
\rho_{f}=K \frac{\rho_{B}}{9} \operatorname{Ln}\left(\frac{\rho_{B}}{\rho_{0}} e^{\frac{34.3}{K}-\frac{\rho_{B}}{\rho_{0}}}\right)
$$

where $K$ is the usual incompressibility modulus and 34.2 (in $\mathrm{fm}^{-1}$ ) was adjusted to fit the usual value of $\rho_{f}$ at $\rho_{0}$. A solution for the spin zero bosons part of stability equation is given by:

$$
\left(T r|\bar{\Sigma}+\bar{\Pi}|^{2}-\tilde{v}_{3}^{2}\right)=\tilde{D} \sqrt{\rho_{B}} \|_{\rho_{B}=\rho_{0}} .
$$

Where the "condensates" of the scalar and pseudo scalar bosons (whenever they occur - such as in [4]) are denoted by $\bar{a}$. The solution above (expression (??))) for the stability equation does satisfy obey the equations of motion. The above expression is, in principle, valid at zero density and at $\rho_{0}$ but seems to be also valid at different $\rho_{B}$. $\tilde{D}$ is a constant to be fixed by this boundary condition [4]. The scalar condensate or classical expectation value, related to the QCD scalar condensate $\bar{\sigma} \propto<\bar{q} q>$, seems to decrease as density increases (from the vaccum) although it may also increase [4] until asymptotic freedom of the underlying theory (QCD) becomes relevant and chiral symmetry would be restored. The remaining equations for the vector fields were discussed extensively in [4].

The averaged value of the Lagrangian yields massive fermions without breaking the flavour symmetry explicitely as long as the three scalar fields $\Sigma_{i i}$, diagonal elements of the matrix $\Sigma$, acquires classical values in the vacuum already. In the medium it yields:

$$
M_{a, b ; s}^{*}=g_{S}<N_{a, s}\left|\left(\bar{\sigma}+\bar{\xi}+i \gamma_{5} \vec{\tau} \cdot \overline{\boldsymbol{\pi}}\right)\right| N_{b, s}>\simeq g_{S} \sum_{a} \bar{\Sigma}_{a, a} \delta_{a, b}
$$

\footnotetext{
${ }^{2} \mathrm{An}$ analogous expression for anti-matter finite density system is defined. In the case of coexistence of both the "chemical equilibrium" must be considered.
} 
In this expression $a, b$ stands for the flavor index $(1,2,3)$. These three scalar condensates would appear due to a spontaneous symmetry breaking of $S U(3) \times S U(3)$ down to $S U(3)$. As the density varies so do the condensates and consequentely the hadronic masses and properties. Experimental value of $E / A, \rho_{0}, K$ and masses are considered for finding other variables. The corresponding results for quark bound system evenutally with hadron-quark mixed states are being investigated.

\section{Matter and Anti-Matter States}

The resulting Dirac equation for the baryons coupled to the classical bosons (the vector fields $V_{\mu}$ and $B_{\mu}^{3}$ scalars and pseudoscalars) with a generalized chemical potential, considered to be included as $\gamma_{\mu} \mu$, is given by:

$$
\left[\gamma_{\mu}\left(\partial^{\mu}-i g_{V} V^{\mu}-i g_{3} B_{3}^{\mu}\right)+\mu \sum_{\mu} \gamma^{\mu}-i g_{s}\left(\bar{\Sigma}+i \gamma_{5} . \bar{\Pi}\right)\right] \Psi(\mathbf{x})=0
$$

Where $B_{3}^{\mu}$ is the contribution of the isovector fields/mesons (the third component) which may develop classical values and are more appropriate for the description of neutron-proton asymmetric nuclear systems including with isospin/flavor asymmetries. This equation has fermions and anti-fermions as solutions with eigenvalues such as $E^{ \pm}=$ $g_{V} V_{0} \pm \sqrt{\mathbf{p}+M^{*}}$, where $M^{*}$ stands for the mass term provenient from the scalar field couplings. If the vector field component $V_{0}$ becomes negative the eingenvalues associated to anti-matter may be favored leading preferentially to anti-matter (bound) states eventually. The solutions associated to these eigenvalues may yield finite baryon density and also finite anti-baryon density. These densities, with the current densities, can be defined as: $\bar{N} \gamma_{\mu} N$ and $N^{T} \gamma_{\mu} \bar{N}^{T}$ with Nambu-Gorkov spinors [5]. However the spectra of the (positive and negative energy) solutions are not the same anymore due to the classical temporal component of the vector field and to the chemical potential. Anti-matter bound states could be considered in the phase diagram of our Universe, constituted rather of matter, as a third axis or the negative extension of the matter density axis (besides density and temperature) eventually associated to the matter density - as to be linear independent or not. This kind of bound state with anti-matter component(s) may occur in relativistic heavy ions collisions under execution and being prepared in RHIC and CERN [6]. These finite density systems may be expected to have relevant dynamical effects. In [5] the effect of classical tensor and vector fields were considered to the formation of superconductive states at very high densities yielding condensates of di-antifermions besides the usual difermions (di-quarks) condensates, ocurring with the breakdown of symmetries such as color among other which would happen also with the consideration of anti-matter fields, as shown above.

\section{Summary}

The linear sigma model with some strange particles for baryons and spin zero and one mesons was considered to describe a finite density hadronic system - below the energies in which deconfinement and chiral/flavor symmetry restora- tion(s) take place. An in medium extended symmetry radius was defined incorporating a strangeness component. Some properties were suggested for the phase diagram of strong interacting systems including regions with new condensates besides the usual scalar QCD condensate. Eventually antimatter bound states may play a significant role in high energy and relativistic heavy ions collisions. The presence of QCD condensates, containing also strangeness degrees of freedom, can produce effects in the dynamics of chiral symmetry restoration - for an effective simplified picture see, for example, reference [3]. Production of strangeness in the (relativistic) heavy ion collisions may be strongly influenced by these condensates because a larger production of strange particles would be favored with the excitation of the condensates containing strange quarks-anti-quarks (eventually in the ground state). Finally, anti-matter formed in the high (relativistic) energy heavy ion collisions may be part of a bound state, although in a small time scale, depending on the value of the vector field coupled to fermions (baryons/quarks) in such energetic environment.

\section{Acknowledgement}

This work has been partially supported by FAPESP, Brazil.

\section{References}

[1] S. Weinberg, The Quantum Theory of Fields -II, Cambridge University Press, (1996); C. Itzykson and J.-B. Zuber, Quantum Field Theory, McGraw Hill Int. Editions, Singapore, (1985).

[2] B.D. Serot and J.D. Walecka, Int. Jour. of Mod. Phys. E6, 515 (1997) and references therein.

[3] F.L. Braghin, Phys. Rev. D64, 125001 (2001), Doctoral thesis, France, 1995/96, unpublished; F.L. Braghin and F.S. Navarra, Phys. Lett. B 508, 243 (2001); F.L.Braghin, Phys. Rev. D57, 3548 (1998).

[4] F.L. Braghin, pré-print IF-1570/2001, Instituto de Física da USP, submitted to publication (2001-2003); F.L. Braghin, under development. 
[5] F.L. Braghin, talk at Journey on Bosons and Gauge symmetries and related subjects, IF-USP, 29th August 2002, without proceedings;

http://www.axpfep1.if.usp.br/finpe/workshop/fabio/transp.html

[6] Proceedings of Quark Matter 2002; International Workshop on Relativistic Aspects of Nuclear Physics, Caraguatatuba, Brazil,
Ed. by T. Kodama et al, World Scientific, (2001).

[7] S. Narison, hep-ph/0009108; C.M. Shakin, Phys. Rev. D65, 114011 (2002).

[8] F.L. Braghin, to be published in Int. Journ. of Modern Phys. D. 